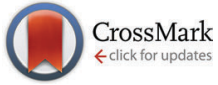

Cite this: Chem. Commun., 2015, 51, 16553

Received 10th July 2015

Accepted 20th September 2015

DOI: $10.1039 / c 5 c c 05726 d$

www.rsc.org/chemcomm

\section{Atomic layer deposition on 2D transition metal chalcogenides: layer dependent reactivity and seeding with organic ad-layers}

\author{
Christian Wirtz, ${ }^{a b}$ Toby Hallam, ${ }^{b}$ Conor Patrick Cullen, ${ }^{a b}$ Nina C. Berner, ${ }^{a b}$ \\ Maria O’Brien, ${ }^{\text {ab }}$ Mario Marcia, ${ }^{c}$ Andreas Hirsch ${ }^{c}$ and Georg S. Duesberg*ab
}

This commmunication presents a study of atomic layer deposition of $\mathrm{Al}_{2} \mathrm{O}_{3}$ on transition metal dichalcogenide (TMD) two-dimensional films which is crucial for use of these promising materials for electronic applications. Deposition of $\mathrm{Al}_{2} \mathrm{O}_{3}$ on pristine chemical vapour deposited $\mathrm{MoS}_{2}$ and $\mathrm{WS}_{2}$ crystals is demonstrated. This deposition is dependent on the number of TMD layers as there is no deposition on pristine monolayers. In addition, we show that it is possible to reliably seed the deposition, even on the monolayer, using non-covalent functionalisation with perylene derivatives as anchor unit.

The integration of transition metal dichalcogenides (TMDs) into existing semiconductor technology is of major interest. ${ }^{1,2}$ The synthesis of these materials has been vastly improved over the last few years and many possible devices have been proposed and realised. ${ }^{3-6}$ But to finally achieve their large-scale integration and production, it is necessary to make TMDs fully CMOS processable. One prerequisite for this is the deposition of subsequent layers on top of the TMD for gating and passivation as the performance and stability of TMD based devices hugely depends on their dielectric environment. This task is nontrivial as any impact on the surface of the TMD will result in the destruction of its electronic properties. It has been shown that encapsulation of the 2D material by mechanical deposition of hexagonal boron nitride results in the best preservation of its electronic properties but this approach is not scalable. ${ }^{7}$

Other frequently used deposition methods for oxides such as sputtering or plasma-enhanced chemical vapour deposition (PECVD) are not suitable as their application will cause damage to the monolayer.

Atomic layer deposition (ALD) is a mild and highly precise technique for thin film deposition, mainly used for depositing

\footnotetext{
${ }^{a}$ School of Chemistry, Trinity College Dublin, Dublin, Ireland. E-mail: duesberg@tcd.ie; Tel: +35318963035

${ }^{b}$ CRANN \& AMBER Institutes, Trinity College, Dublin, Ireland 'Department of Chemistry and Pharmacy, Organische Chemie II, Friedrich-Alexander Universität Erlangen-Nürnberg, Henkestrasse 42, 91054 Erlangen, Germany
}

gate oxides in integrated circuits. ${ }^{8}$ The most common ALD process is the deposition of $\mathrm{Al}_{2} \mathrm{O}_{3}$ from alternating exposures of trimethylaluminium (TMA, $\left.\mathrm{Al}\left(\mathrm{CH}_{3}\right)_{3}\right)$ and water according to the reaction: ${ }^{9}$

$$
2 \mathrm{Al}\left(\mathrm{CH}_{3}\right)_{3}+3 \mathrm{H}_{2} \mathrm{O} \rightarrow \mathrm{Al}_{2} \mathrm{O}_{3}+6 \mathrm{CH}_{4} \quad \Delta H=-376 \mathrm{kcal}
$$

This reaction is thermodynamically highly favourable and works over a large range of temperatures with temperatures between $33{ }^{\circ} \mathrm{C}$ and $500{ }^{\circ} \mathrm{C}$ demonstrated, making it very reliable and common in the silicon and III-V semiconductor industries. ${ }^{10,11}$ However, in the initial step the TMA needs a surface hydroxyl group with which it reacts and the lack of such groups on the TMD's basal plane makes starting the deposition non-trivial; a challenge also encountered with graphene. ${ }^{12-15}$ Using ozone instead of water may prove harmful to the oxidation-sensitive TMD layers, though there have been some recent successes. ${ }^{16}$ An initial, purely adsorptionbased deposition can be achieved but tends to be dependent on temperature and other factors like underlying electronic structure and is therefore often not entirely reproducible; it has been shown several times that studies may not reproduce results under apparently similar conditions. ${ }^{12,17,18}$

In this study we used single crystalline layers of $\mathrm{MoS}_{2}$ and $\mathrm{WS}_{2}$ grown via chemical vapour deposition (CVD) as previously demonstrated. ${ }^{19}$ The ALD on those layers was performed at the relatively low temperature of $80{ }^{\circ} \mathrm{C}$ with 27 cycles of TMA and $\mathrm{H}_{2} \mathrm{O}$ using 0.5 second pulses with 20 seconds purge time $\left(60 \mathrm{sccm} \mathrm{N}_{2}\right.$ ) and should yield $\sim 3 \mathrm{~nm}$ of $\mathrm{Al}_{2} \mathrm{O}_{3}$. Those samples were analysed with atomic force microscopy (AFM) to determine step heights and scanning Raman spectroscopy. $\mathrm{Al}_{2} \mathrm{O}_{3}$ as deposited by ALD does not exhibit a Raman signal but both $\mathrm{MoS}_{2}$ and $\mathrm{WS}_{2}$ have characteristic peaks in the region from $370 \mathrm{~cm}^{-1}$ to $420 \mathrm{~cm}^{-1}$.

The $\mathrm{MoS}_{2}$ and $\mathrm{WS}_{2}$ layers have the characteristic triangular shape and consisted of mostly monolayer except for the flake centre which occasionally exhibited an onset of multilayer growth. This can be seen in the different contrast regions shown in Fig. 1a. An AFM scan (Fig. 1b), along with the line 

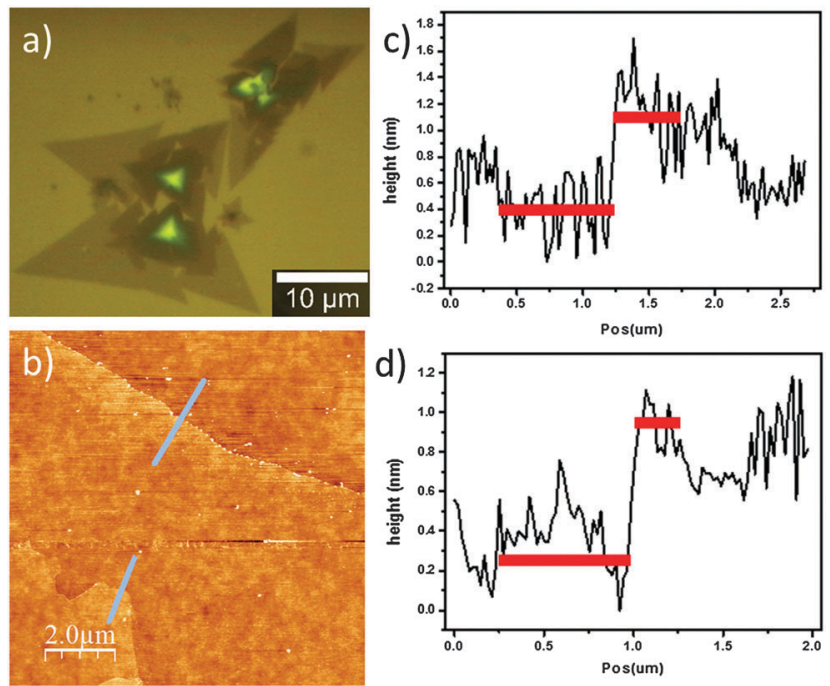

Fig. 1 Representation of the $\mathrm{MoS}_{2}$ flakes used in the experiment: (a) optical image of flakes consisting of crystalline monolayers and some multilayers in the flake centres. (b) AFM image of a $\mathrm{MoS}_{2}$ flake; (c and d) show the line profiles along the marked lines in (b) with a step height of $0.7 \mathrm{~nm}$.

scans in Fig. 1c and d, shows the ideal step height of $0.7 \mathrm{~nm}$ per TMD layer. This proved to be very useful as it allowed for side-byside comparison of $\mathrm{Al}_{2} \mathrm{O}_{3}$ ALD on mono- and bilayer samples.

In Fig. 2a we present an AFM image of a triangular $\mathrm{WS}_{2}$ flake on $\mathrm{SiO}_{2}$ after $3 \mathrm{~nm} \mathrm{Al}_{2} \mathrm{O}_{3}$ deposition. It is apparent that the triangular flake (dark region) is lower than the surrounding substrate. This becomes better visible in the line profile across the flake as shown in Fig. $2 \mathrm{~b}$. The step height between the flake and substrate is $\sim 2.3 \mathrm{~nm}$ after deposition, perfectly corresponding to the difference of $3 \mathrm{~nm} \mathrm{Al}{ }_{2} \mathrm{O}_{3}$ deposition minus the flake height of $0.7 \mathrm{~nm}$. Thus there is no deposition of $\mathrm{Al}_{2} \mathrm{O}_{3}$ on the monolayer. The double-layered centre of the flake increased in height to $\sim 3.7 \mathrm{~nm}$ with respect to the monolayer, perfectly corresponding to $0.7 \mathrm{~nm}$ flake height plus $3 \mathrm{~nm} \mathrm{Al}_{2} \mathrm{O}_{3}$. At the edge of the monolayer $\mathrm{Al}_{2} \mathrm{O}_{3}$ was deposited which we attribute to dangling bonds and defects that are more reactive. This high selectivity in the deposition process was found in both both $\mathrm{WS}_{2}$ and $\mathrm{MoS}_{2}$ single crystals grown via CVD. This is to our knowledge the first observation of selective chemical behaviour between mono- and doublelayered or multilayered TMDs. We attribute this selectivity to two factors: firstly, our films must be extremely clean with no defects in the basal plane as otherwise the ALD would occur at those centres like at the flake edge. As the films came straight out of the oven and there was no transfer, no polymer residue could seed any ALD, something that may be responsible for the inconsistent results in the field so far. Secondly, TMDs undergo a significant change in electronic structure when going from multi-layered structures to single layers. ${ }^{20,21}$ There is a change from indirect bandgap of $1.2 \mathrm{eV}$ and $1.3 \mathrm{eV}$ to a direct bandgap of $1.9 \mathrm{eV}$ and $2 \mathrm{eV}$ for $\mathrm{MoS}_{2}$ and $\mathrm{WS}_{2}$, respectively. ${ }^{22,23}$ Hence the initially adsorption-based ALD may be so heavily influenced by this difference in underlying electronic structure that it is significantly different for monolayers in comparison to bulk. However, theoretical modelling is required a)

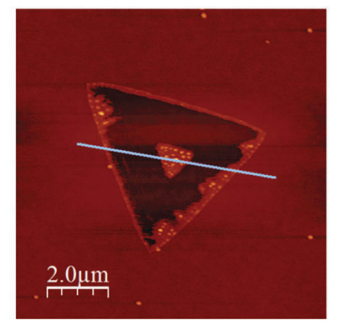

b)

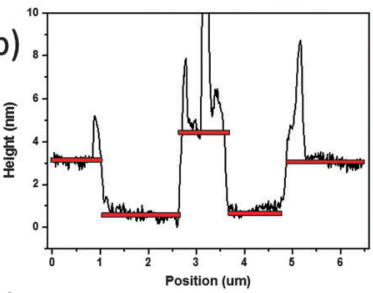

c)

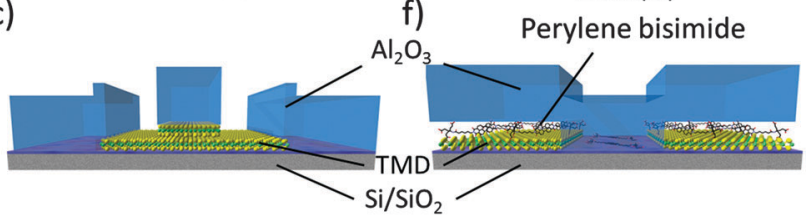

d)
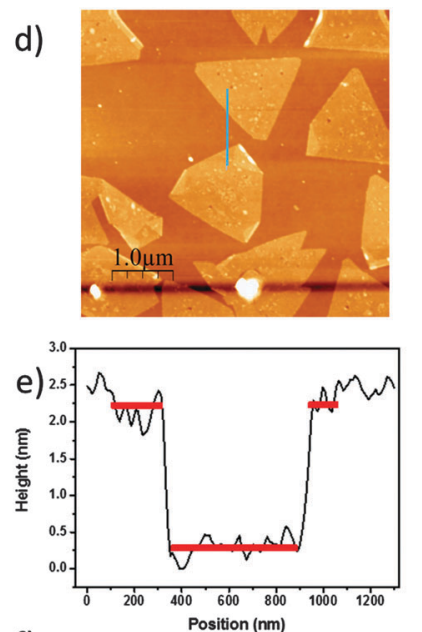

f)

()
Fig. 2 (a) AFM analysis of TMDs after ALD. (a) Topography of a WS 2 triangle after 27 cycles of TMA and $\mathrm{H}_{2} \mathrm{O}$. The monolayer part of the triangle lies lower than the surrounding $\mathrm{SiO}_{2}$. (b) Line profile along the marked line in (a). The step height between monolayer $\mathrm{WS}_{2}$ and $\mathrm{SiO}_{2}$ is $2.3 \mathrm{~nm}$ and between monolayer $W_{2}$ and bilayer $W_{2}$ is $3.7 \mathrm{~nm}$. Only the monolayer edges were covered with the expected $3 \mathrm{~nm} \mathrm{Al}_{2} \mathrm{O}_{3}$. (c) Schematic representation of the surface structure in (a) as indicated by the AFM scan. (d) Topography of $\mathrm{MoS}_{2}$ triangles after 27 cycles of ALD after seeding with a perylene bisimide derivative. The flakes are higher than the surrounding substrate. (b) Line profile along the line marked in (d). The step height is $1.9 \mathrm{~nm}$. (f) Schematic representation of the structure of (d) as indicated by the scan.

to further investigate the underlying mechanism. This selectivity can be a significant advantage: covering all layers but the monolayer with a dielectric leaves only the material with the direct bandgap exposed. This provides a novel pathway to select monolayer regions and can be used for vertical device fabrication and selective chemistry.

However, deposition of uniform dielectrics on monolayer TMDs is desired for passivation and electronic device fabrication. We therefore explore non-covalent functionalisation of TMD basal planes with molecules that contain $-\mathrm{OH}$ and $-\mathrm{COOH}$ units which can react with TMA and thereby seed the reaction. ${ }^{8,24}$

We use a perylene-based anchor unit on our TMDs. ${ }^{25}$ This perlyene, shown in Fig. 3a, has a large aromatic core that can non-covalently attach to the TMD and end-groups with hydroxy and carboxyl functionalities. It is similar to molecules that have been employed to seed these depositions on graphene by Alaboson et al. with the difference that our molecule has longer end-group chains with carboxylic acid groups. ${ }^{26}$ This kind of end-group has been shown to seed $\mathrm{Al}_{2} \mathrm{O}_{3}$ ALD from TMA and $\mathrm{H}_{2} \mathrm{O}^{27}$ The perylene adsorbs very strongly on TMDs and is deposited from an aqueous $\mathrm{pH} 7$ buffer solution by simple dipcoating. Washing the sample with water after deposition leaves only a thin layer of perylenes on $\mathrm{MoS}_{2}$ and $\mathrm{WS}_{2}$. As can be seen in the AFM scan in Fig. 2d, ALD on a perylene-covered sample 
a)
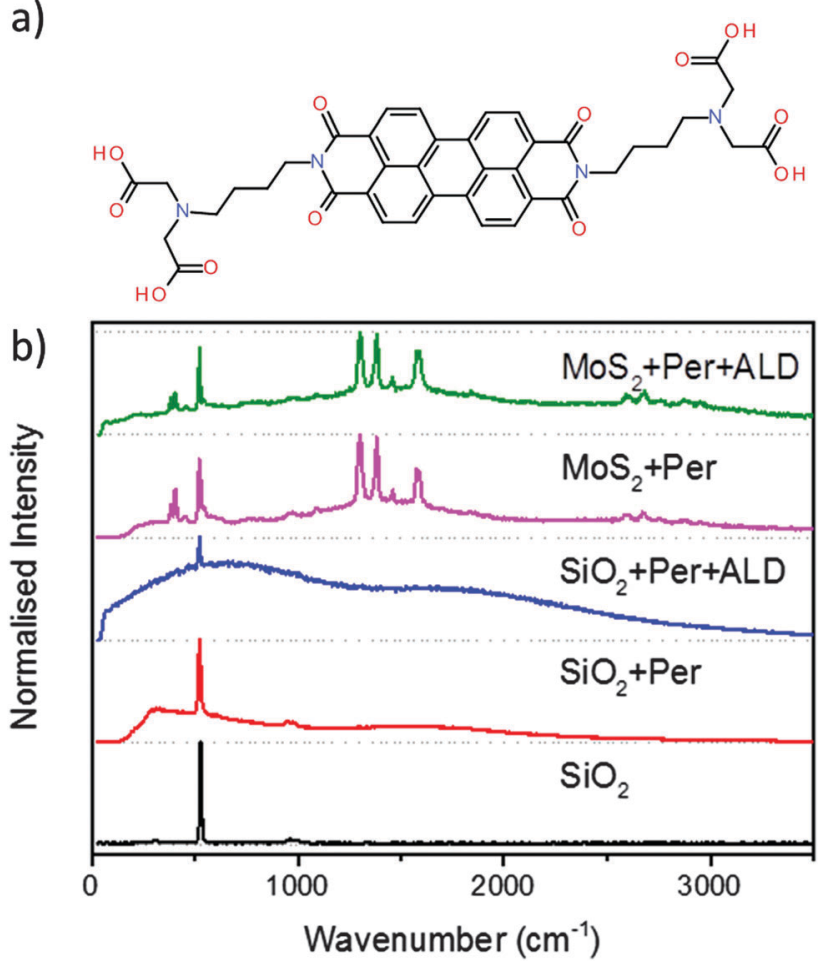

Fig. 3 (a) Chemical structure of the perylene bisimide used for non-covalent functionalisation and ALD seeding. (b) Normalised Raman spectra of the samples at various stages: the $\mathrm{SiO}_{2}$ spectrum only shows the standard strong peak at $521 \mathrm{~cm}^{-1}$. Upon functionalisation with perylene bisimide a strong fluorescent background is observed. This remains after ALD. On the TMD the perylene shows several intense peaks in the region of $1300 \mathrm{~cm}^{-1}$ to $1600 \mathrm{~cm}^{-1}$ and around $2700 \mathrm{~cm}^{-1}$ while the $\mathrm{MoS}_{2}$ has its peaks at $4400 \mathrm{~cm}^{-1}$. All these peaks remain after ALD

leads to a perfectly homogeneous deposition of $\mathrm{Al}_{2} \mathrm{O}_{3}$ on all layers of $\mathrm{MoS}_{2}$. The topological cross-section in Fig. 2e reveals that the step height between $\mathrm{MoS}_{2}$ flake and $\mathrm{SiO}_{2}$ substrate is not $0.7 \mathrm{~nm}$ any more but around $1.9 \mathrm{~nm}$. The additional height difference of $1.2 \mathrm{~nm}$ implies that the perylene adsorbs better on the TMDs than on $\mathrm{SiO}_{2}$ and that it may be in an upright conformation rather than laying flat.

To investigate the effect of ALD on the TMD we utilised scanning Raman spectroscopy. The Raman spectra of both $\mathrm{MoS}_{2}$ and $\mathrm{WS}_{2}$ are very similar, with two peaks closely spaced in the region around $400 \mathrm{~cm}^{-1}$, shown for $\mathrm{MoS}_{2}$ in Fig. 4d. Change in molecular or electronic structure due to $\mathrm{Al}_{2} \mathrm{O}_{3}$ deposition should result in an alteration of those signals. ${ }^{28}$ The out-of-plane Raman active vibration of $\mathrm{MoS}_{2}$ at $405-410 \mathrm{~cm}^{-1}$ has previously been shown to shift with layer number and doping. ${ }^{29} \mathrm{~A}$ shift with layer number is expectedly observed as shown in Fig. 4a but no further shift upon $\mathrm{Al}_{2} \mathrm{O}_{3}$ deposition occurs as shown in Fig. $4 \mathrm{~b}$. Hence the electronic structure of the multilayer (coated) and monolayer (uncoated) TMD appears unperturbed upon $\mathrm{Al}_{2} \mathrm{O}_{3}$ deposition.

Raman mapping of the TMD's "A" peak (Fig. 4c) shows that the $\mathrm{MoS}_{2}$ and $\mathrm{WS}_{2}$ are not damaged by the deposition and retain their characteristics although there is a minor blueshift of $\sim 1 \mathrm{~cm}^{-1}$, potentially indicating p-doping. ${ }^{24,30}$ As this is at our spectrometer's resolution limit it will require further investigation.

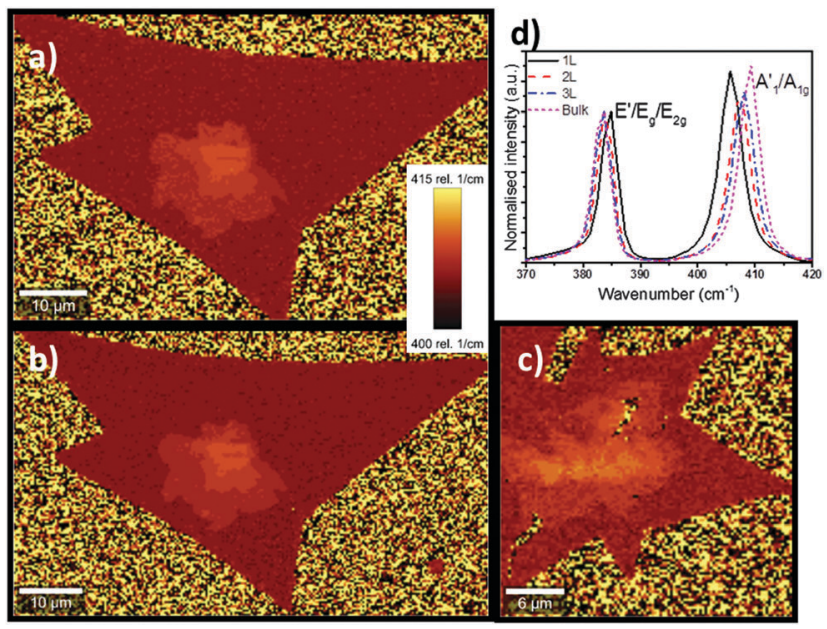

Fig. 4 Mapped out Raman spectrographs of $\mathrm{MoS}_{2}$ flakes. Shown is the $\mathrm{MoS}_{2} \mathrm{~A} 1 \mathrm{~g}$ peak position whose shift indicates different layer numbers and doping levels. (a) Pristine $\mathrm{MoS}_{2}$, the central area of the flake shows multilayer signal; (b) the same $\mathrm{MoS}_{2}$ flake after exposure to 27 cycles of TMA and $\mathrm{H}_{2} \mathrm{O}$, yielding $3 \mathrm{~nm}$ thick $\mathrm{Al}_{2} \mathrm{O}_{3}$; there is no appreciable change, indicating the exposure had no impact on electronic structure. (c) A different $\mathrm{MoS}_{2}$ flake after perylene deposition and exposure to ALD shows a slight blueshift, possibly indicating some p-doping. (d) Average spectra of the different regions of the flake in (a).

Investigation of the perylene bisimide's Raman spectrum, shown in Fig. 3b, shows several features. On just $\mathrm{SiO}_{2}$ it has a very strong fluorescent background and does not show any features. After ALD this fluorescence remains, indicating the continued presence of the perylene. On TMDs it shows several peaks in the regions around $1500 \mathrm{~cm}^{-1}$ and $2700 \mathrm{~cm}^{-1}$ which remain after ALD, confirming its presence at all stages. This makes the interpretation of the $1.9 \mathrm{~nm}$ step height from Fig. 2e difficult and indicates different molecular orientations on $\mathrm{SiO}_{2}$ and $\mathrm{MoS}_{2}$.

In summary, we demonstrated that there exists selective chemistry of monolayer TMD surfaces over their multilayer counterparts. We have shown that this can lead to the selective deposition of $\mathrm{Al}_{2} \mathrm{O}_{3}$ which is essentially a method to selectively mask all areas but monolayer of $\mathrm{MoS}_{2}$ and $\mathrm{WS}_{2}$. This will hopefully lead to a whole variety of layer number selective chemistry in future. Furthermore we demonstrated that an easily accessible, non covalent functionalisation with perylene derivatives allows for reliable $\mathrm{ALD}$ of $\mathrm{Al}_{2} \mathrm{O}_{3}$ on monolayer TMDs without damage to their electronic integrity as observed by Raman spectroscopy. These findings are an important step forward toward integration of TMDs in real devices.

The authors thank Science Foundation Ireland for funding under the grant SFI-Pica:Pi_10/IN.1/I3030.

\section{References}

1 D. Jariwala, V. K. Sangwan, L. J. Lauhon, T. J. Marks and M. C. Hersam, ACS Nano, 2014, 8, 1102-1120.

2 A. C. Ferrari, F. Bonaccorso, V. Fal'ko, K. S. Novoselov, S. Roche, P. Boggild, S. Borini, F. H. L. Koppens, V. Palermo, N. Pugno, J. A. Garrido, R. Sordan, A. Bianco, L. Ballerini, M. Prato, E. Lidorikis, J. Kivioja, C. Marinelli, T. Ryhanen, A. Morpurgo, J. N. Coleman, 
V. Nicolosi, L. Colombo, A. Fert, M. Garcia-Hernandez, A. Bachtold, G. F. Schneider, F. Guinea, C. Dekker, M. Barbone, Z. Sun, C. Galiotis, A. N. Grigorenko, G. Konstantatos, A. Kis, M. Katsnelson, L. Vandersypen, A. Loiseau, V. Morandi, D. Neumaier, E. Treossi, V. Pellegrini, M. Polini, A. Tredicucci, G. M. Williams, B. Hee Hong, J.-H. Ahn, J. Min Kim, H. Zirath, B. J. van Wees, H. van der Zant, L. Occhipinti, A. Di Matteo, I. A. Kinloch, T. Seyller, E. Quesnel, X. Feng, K. Teo, N. Rupesinghe, P. Hakonen, S. R. T. Neil, Q. Tannock, T. Lofwander and J. Kinaret, Nanoscale, 2015, 7, 4598-4810.

3 H. R. Gutiérrez, N. Perea-López, A. L. Elías, A. Berkdemir, B. Wang, R. Lv, F. López-Urías, V. H. Crespi, H. Terrones and M. Terrones, Nano Lett., 2013, 13, 3447-3454.

4 Y. Gong, J. Lin, X. Wang, G. Shi, S. Lei, Z. Lin, X. Zou, G. Ye, R. Vajtai, B. I. Yakobson, H. Terrones, M. Terrones, B. Tay, J. Lou, S. T. Pantelides, Z. Liu, W. Zhou and P. M. Ajayan, Nat. Mater., 2014, 13, 1135-1142.

5 X. Duan, C. Wang, J. C. Shaw, R. Cheng, Y. Chen, H. Li, X. Wu, Y. Tang, Q. Zhang, A. Pan, J. Jiang, R. Yu, Y. Huang and X. Duan, Nat. Nanotechnol., 2014, 9, 1024-1030.

6 A. M. van der Zande, P. Y. Huang, D. A. Chenet, T. C. Berkelbach, Y. You, G.-H. Lee, T. F. Heinz, D. R. Reichman, D. A. Muller and J. C. Hone, Nat. Mater., 2013, 12, 554-561.

7 T. Roy, M. Tosun, J. S. Kang, A. B. Sachid, S. B. Desai, M. Hettick, C. C. Hu and A. Javey, ACS Nano, 2014, 8, 6259-6264.

8 S. M. George, Chem. Rev., 2010, 110, 111-131.

9 R. L. Puurunen, J. Appl. Phys., 2005, 97, 121301.

10 M. D. Groner, F. H. Fabreguette, J. W. Elam and S. M. George, Chem. Mater., 2004, 16, 639-645.

11 R. Matero, A. Rahtu, M. Ritala, M. Leskelä and T. Sajavaara, Thin Solid Films, 2000, 368, 1-7.

12 H. Liu, K. Xu, X. J. Zhang and P. D. Ye, Appl. Phys. Lett., 2012, 100, 152115.

13 B. Dlubak, P. R. Kidambi, R. S. Weatherup, S. Hofmann and J. Robertson, Appl. Phys. Lett., 2012, 100, 173113.

14 J. Kim and S. Jandhyala, Thin Solid Films, 2013, 546, 85-93.
15 S. McDonnell, B. Brennan, A. Azcatl, N. Lu, H. Dong, C. Buie, J. Kim, C. L. Hinkle, M. J. Kim and R. M. Wallace, ACS Nano, 2013, 7, 10354-10361.

16 L. X. Cheng, X. Y. Qin, A. T. Lucero, A. Azcatl, J. Huang, R. M. Wallace, K. Cho and J. Kim, ACS Appl. Mater. Interfaces, 2014, 6, 11834-11838.

17 S. Brunauer, P. H. Emmett and E. Teller, J. Am. Chem. Soc., 1938, 60, 309-319.

18 J. Yang, S. Kim, W. Choi, S. H. Park, Y. Jung, M. H. Cho and H. Kim, ACS Appl. Mater. Interfaces, 2013, 5, 4739-4744.

19 M. O'Brien, N. McEvoy, T. Hallam, H.-Y. Kim, N. C. Berner, D. Hanlon, K. Lee, J. N. Coleman and G. S. Duesberg, Sci. Rep., 2014, 4, 7374.

20 K. F. Mak, C. Lee, J. Hone, J. Shan and T. F. Heinz, Phys. Rev. Lett., 2010, 105, 136805.

21 W. Zhao, Z. Ghorannevis, L. Chu, M. Toh, C. Kloc, P.-H. Tan and G. Eda, ACS Nano, 2013, 7, 791-797.

22 A. M. Goldberg, A. R. Beal, F. A. Lévy and E. A. Davis, Philos. Mag., 1975, 32, 367-378.

23 A. L. Elías, N. Perea-López, A. Castro-Beltrán, A. Berkdemir, R. Lv, S. Feng, A. D. Long, T. Hayashi, Y. A. Kim, M. Endo, H. R. Gutiérrez, N. R. Pradhan, L. Balicas, T. E. Mallouk, F. López-Urías, H. Terrones and M. Terrones, ACS Nano, 2013, 7, 5235-5242.

24 X. R. Wang, S. M. Tabakman and H. J. Dai, J. Am. Chem. Soc., 2008, 130, 8152-8153.

25 M. Marcia, P. Singh, F. Hauke, M. Maggini and A. Hirsch, Org. Biomol. Chem., 2014, 12, 7045-7058.

26 J. M. P. Alaboson, Q. H. Wang, J. D. Emery, A. L. Lipson, M. J. Bedzyk, J. W. Elam, M. J. Pellin and M. C. Hersam, ACS Nano, 2011, 5, 5223-5232.

27 M. Li, M. Dai and Y. J. Chabal, Langmuir, 2009, 25, 1911-1914.

28 B. Chakraborty, A. Bera, D. V. S. Muthu, S. Bhowmick, U. V. Waghmare and A. K. Sood, Phys. Rev. B: Condens. Matter Mater. Phys., 2012, 85, 161403.

29 H. Li, Q. Zhang, C. C. R. Yap, B. K. Tay, T. H. T. Edwin, A. Olivier and D. Baillargeat, Adv. Funct. Mater., 2012, 22, 1385-1390.

30 Y. Shi, J.-K. Huang, L. Jin, Y.-T. Hsu, S. F. Yu, L.-J. Li and H. Y. Yang, Sci. Rep., 2013, 3, 1839. 